\title{
Spectral X-ray ptychography for the investigation of technical catalysts
}

\author{
A. Kulow ${ }^{1}$, S. Ould-Chikh², R. Boudjehem ${ }^{1}$, J.-L. Hazemann ${ }^{1}$, T. Shoinkhorova ${ }^{2}$, A. Dikhtiarenko², M.A. Alabdullah², \\ J. Gascon ${ }^{2}$, R. Sougrat ${ }^{3}$, J. C. da Silva ${ }^{1}$
}

\begin{abstract}
${ }^{1}$ Univ. Grenoble Alpes, CNRS, Grenoble INP, Institut Néel, 38000 Grenoble, France, ${ }^{2}$ King Abdullah University of Science and Technology, KAUST Catalysis Center, Advanced Functional Materials, Thuwal 23955, Saudi Arabia, ${ }^{3}$ King Abdullah University of Science and Technology, Core Labs, Thuwal 23955, Saudi Arabia
\end{abstract}

\author{
anico.kulow@neel.cnrs.fr
}

Catalysts are ubiquitous materials that play a major role in many areas of economy and everyday life. The development and study of catalysts is important for progress in areas such as environment, energy, and fuels, with the main goal being to improve the performance and efficiency of catalysts, especially at the industrial scale. Therefore, a thorough analysis is crucial to understand the relation between structure and performance, the deactivation process and the reasons for the loss of efficiency over the lifetime. This analysis is challenging, because technical catalysts are complex multicomponent bodies, ranging from dozens of $\mu \mathrm{m}$ to several $\mathrm{cm}$, consisting of active phases, supports and additives in shaped forms suitable for their application. One of the most important conversion processes in petroleum refineries is Fluid Catalytic Cracking (FCC) in which heavy hydrocarbon fractions of crude oil are converted into valuable products such as olefins and aromatics [1]. For this process, FCC particles of $50-100 \mu \mathrm{m}$ diameter are used in an up-flow reactor, where they move up, whereas the feed flows downward. During the short contact time, catalyst and feed can react. During this reaction the catalyst is partially deactivated by coke formed during the cracking and a subsequent regeneration cycle is required [2]. Thus, the characterization of the microstructure at different length scales with a spatial resolution at the nanometer length scale and a large field of view is necessary, but also the investigation of the location and chemical state of the active metallic sites in the structure.

The imaging of a large field of view with a resolution of $\sim 30-100 \mathrm{~nm}$ is possible with ptychography, even for low absorbing samples. To get spatial resolved spectral information, spectro-ptychography can be used, where the measurements are repeated at different energies, including the absorption edge of a specific element. This method has already been applied for the nanoscale chemical imaging and structural analysis of a heterogeneous catalyst [3].
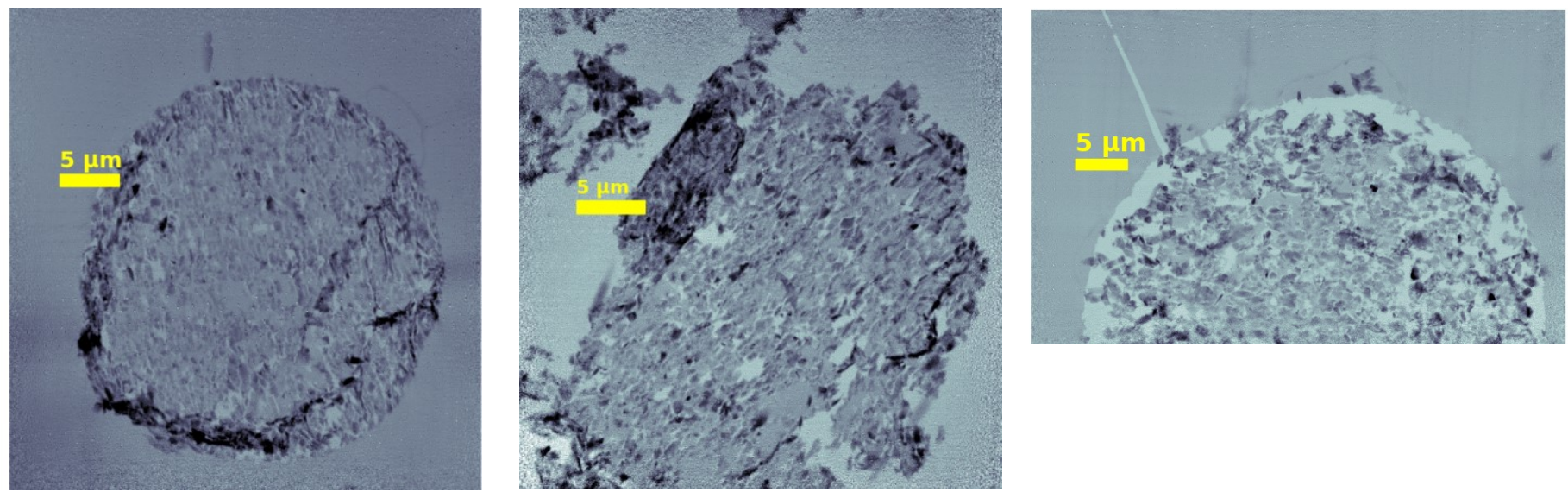

Figure 1 : Reconstructed phase images of a FCC particle at different lifetimes. Left: fresh; middle: spent; right: regenerated.

We investigated a FCC catalyst containing $10 \mathrm{wt} . \% \mathrm{Mn}_{2} \mathrm{O}_{3}$ at different lifetimes by means of spectro-ptychography. Ptychographic scans are repeated at 40 different energies around the $\mathrm{Mn} \mathrm{K}$-edge. We show here the results of the experiment carried out at the beamline ID16B at ESRF, where this method has never been used before. The absorption is weak due to the low concentration of Mn and the small thickness of the samples, and hence we work with the phase contrast images. The phase contrast can be associated with the anomalous scattering factor $\mathrm{f}^{\prime}$, which is energy dependent in the proximity of absorption edges. The f' spectra can be extracted by comparing the reconstructed phase contrast images recorded at different energies. The work includes the preparation of the instrumentation, the development of the algorithms for the data preparation and the python programs for the spectral analysis. We show the methodological developments necessary for the extraction of the information from the obtained measurements, starting from the phase retrieval and normalization of the phase images, to the alignment of the images of different energies, to the extraction of the f' spectra and the search for the Mn signature in the sample.

[1] W. Letzsch,Handbook of Petroleum Processing, Springer Int. Publishing, Cham 2015, 216-316.

[2] A. Corma, et al., Catalysis Science \& Technology 2017, 7, 12.

[3] M. Hirose, et al., Angew. Chem. Int. Ed. 2017, 56, 1-6.

Keyword : technical catalyst ; fluid catalytic cracking (FCC) ; X-ray ptychography ; spectro-ptychography 